\title{
Dynamic Analysis of Traffic State and Congestion Propagation on Bidirectional Grid Network
}

\author{
Shu-bin Li, ${ }^{1}$ Bai-bai Fu, ${ }^{2}$ and Jian-feng Zheng $^{3}$ \\ ${ }^{1}$ Public Security Department, Shandong Police College, Jinan 250014, China \\ ${ }^{2}$ School of Architecture and Urban Planning, Shandong Jianzhu University, Jinan 250101, China \\ ${ }^{3}$ MOE Key Laboratory for Urban Transportation Complex Systems Theory and Technology, \\ Beijing Jiaotong University, Beijing 100044, China \\ Correspondence should be addressed to Bai-bai Fu; fubaibai@163.com
}

Received 12 July 2013; Revised 22 October 2013; Accepted 29 October 2013

Academic Editor: Wuhong Wang

Copyright (C) 2013 Shu-bin Li et al. This is an open access article distributed under the Creative Commons Attribution License, which permits unrestricted use, distribution, and reproduction in any medium, provided the original work is properly cited.

\begin{abstract}
Many traffic problems in China such as traffic jams and air pollutions are mainly caused by the increasing traffic volume. In order to alleviate the traffic congestion and improve the network performance, the analysis of traffic state and congestion propagation has attracted a great interest. In this paper, an improved mesoscopic traffic flow model is proposed to capture the speed-density relationship on segments, the length of queue, the flow on links, and so forth, The self-developed dynamic traffic simulation software (DynaCHINA) is used to reproduce the traffic congestion and propagation in a bidirectional grid network for different demand levels. The simulation results show that the proposed model and method are capable of capturing the real traffic states. Hence, our results can provide decision supports for the urban traffic management and planning.
\end{abstract}

\section{Introduction}

During the past decades, researchers in operations research, transportation engineering, and computer science have taken a keen interest in exploring the problem of traffic congestion and propagation in transportation systems. From the macroview, the congestion formation on a single bottleneck was studied [1-3] and the effect of congestion in a traffic network at the macroscopic system level was reported [4-6]. Particularly, Gentile et al. [5] proposed a new model for the within-day Dynamic Traffic Assignment (DTA) on road networks where the simulation of queue spillovers was explicitly addressed, and the efficiency of the proposed algorithm and its applicability to real instances with large-scale networks was evaluated. At the microscopic system level, there were only a few studies on congestion propagation phenomenon, including the reliability analysis of pedestrian [7] and carfollowing safety model [8]. Long et al. $[9,10]$ and Zhang et al. [11] used the cell transmission model to simulate the formation and dissipation of traffic congestion. It was very difficult to analyze the congestion propagation by using the analytical models. This is because the dynamic propagation of traffic flow in network is extremely complicated, which is related to human behavior (e.g., departure time choice, route choice) and network structure. Therefore, many researchers explore the congestion propagation by making use of the simulation methods. Roberg $[12,13]$ proposed simulation models and a number of strategies which could be exploited to achieve a controlled dispersion of traffic jams. Roberg and Abbess [14] applied a simulation model to investigate the diagnosis and treatment of traffic jams. Roberg-Orenstein et al. [15] developed several alternative strategies to avoid gridlock and dissipate traffic jams, in terms of the installation of bans at specific network locations. Wright and Roberg [16] proposed a simple analytical model for incident-based jam growth and discussed the effect of the length of the channelized part of roads and stop-line width assignment on jam formation. Wright and Roberg-Orenstein [17] developed simple models for traffic jams and strategies for congestion control on idealized rectangular grid networks.

Most of the mentioned works mainly focus on dynamic of traffic flow, and the congestion propagation characteristics at different demand levels have not been taken into consideration. As we know, several simulation-based DTA systems, 
including DynaMIT [18] and DynaSMART-X [19], have been developed for a wide range of Intelligent Transportation System (ITS) applications, and can be used to solve this problem. Unfortunately, these systems cannot be deployed directly in China because of some local traffic characteristics, such as mixed traffic flow situations and trip-maker behavior. In this paper, Dynamic Consistent Hybrid Information based on Network Assignment (DynaCHINA) is used, and the authors are the main member for developing this software.

The paper will be organized as follows. Section 1 introduces the background of the problem and some related studies. Section 2 is the overview of the software DynaCHINA. Section 3 gives dynamic of vehicle movement. Section 4 shows the numerical examples. Section 5 is the conclusion remarks.

\section{Overview of DynaCHINA}

DynaCHINA is a simulation-based real time DTA system that estimates and predicts the current and future traffic conditions. In order to guarantee the credibility of the information to travelers, the guidance provided by DynaCHINA is consistent. Based on the expected response to information, DynaCHINA simulates the traffic conditions that will most likely be experienced by travelers. The historical data, realtime surveillance data, and expected traffic control settings are input data. DynaCHINA can generate prediction-based guidance with respect to departure time, pretrip route and mode choice decisions, and en-route route choice decisions. It supports both prescriptive and descriptive information [18]. Hybrid information means that DynaCHINA optimally uses various data sources (historical, surveillance, O-D data, and floating car data) and hybrid optimization techniques to generate reliable traffic predictions and travel guidance information (such as departure time, mode, and route recommendations) for a variety of information systems and information dissemination strategies in real time. The information provided by DynaCHINA is generated on the basis of dynamic network assignment.

The performance of a simulation-based real-time traffic estimation and prediction system (TrEPS) depends on traffic simulation model which realistically captures vehicular traffic flow dynamics. The origin-destination (OD) flow estimation and prediction methodology incorporate any available information from conventional and emerging traffic data collection technologies (such as automatic vehicle identification (AVI) systems and probe vehicles). In order to address the traffic problems in China, DynaCHINA is different from DynaMIT and DynaSMART, as shown below.

(1) Anisotropic supply simulator: DynaCHINA uses two main simulation tools, that is, the demand simulator and the supply simulator. The demand simulator is a microscopic simulator that mainly deals with the estimation and prediction of time-dependent OD flows, demand disaggregation to model the socioeconomic characteristics of drivers, and their decisions of departure time and route choice using behavioral models. The supply simulator is a mesoscopic traffic simulator which is used to simulate vehicular movement on the given network. It can be used to infer traffic flows, queue lengths, speeds, travel times, and densities at all points on the network and thereby serve to indicate network performance [6]. To handle the queue formation and discharge processes, DynaMIT treats moving and queued portions of a link separately, while vehicles in the free-moving portion of the link are moved according to a user-defined macroscopic speed-density relationship. DynaSMART uses virtual queue in conjunction with minimal positive speed. Queue modeling approach in DynaMIT may be conceptually contrived and it is difficult for proper implementation because defining distinct free-moving and queue portions of the traffic stream in general traffic conditions is nontrivial. Transient queues or shockwaves caused by the temporary flow rate transitions may be detected, unless a highly complex set of rules is devised. In DynaSMART, the accuracy of the resultant travel time and link penalty estimation is questionable. Anisotropic mesoscopic model addresses the aforementioned anisotropic property, vehicle moving, and queue formation and discharging in an intuitive and unified manner.

(2) Availability of floating car data (FCD): both DynaMIT and DynaSMART use the loop detector data to calibrate the model parameters and validate their estimation and prediction capabilities. In the framework for the calibration and validation of DynaCHINA, FCD of 60,000 floating cars are available, in addition to the loop detector data and camera data in Beijing. In case of a positive validation, FCD could make it possible to get the accurate traffic information and travel times on roads where the loops and cameras are missing or out of function. The use of the car itself as a floating traffic sensor is a viable additional information source. FCD is well known as an excellent source of high-quality traffic content. FCD is not a service by itself and it needs to be bundled with other services in an intelligent way (e.g., hybrid navigation). The researchers of DynaCHINA team use the advanced data fusion technologies to integrate and process data from different sources. For example, travel time data from FCD will help calculate route choice probabilities and get more accurate assignment matrix, even set moving speed for some road segments directly instead of using speed-density relationship. DynaCHINA team has the exclusive permission to access FCD in Beijing. Incorporating FCD in the OD estimation problem will improve the effectiveness of the dynamic traffic demand prediction models [19].

(3) Modeling mixed traffic flow including bicycles, motorcycles, and pedestrians: in China, the mixedtraffic streams in urban networks are composed of motorized, nonmotorized vehicles and pedestrians. These streams comprise standard vehicles, such as cars, buses, and trucks, nonstandard vehicles such as bicycles, motorcycles, and other refitted vehicles as well as pedestrians. For the nonstandard vehicles, 
they are slightly restricted by codes, road conditions, and technique criterions. Meanwhile, their traveling objective and routes are even more flexible. Not only there are more differences among the characteristics of each nonstandard vehicle, but also their driver behaviors are obviously diverse from the standard vehicles $[20,21]$. At present, there rarely exist models suitable for the analysis of traffic streams with nonstandard vehicles, since the most available models are limited in scope and effectiveness. Inevitably, those existent approaches require improvement. The characteristics and special driver behaviors of nonstandard vehicles in the mixed traffic streams are modeled exogenously, which can help calibrate the model parameters of DynaCHINA, realistically and effectively. Based on Cellular Automation (CA) model and fuzzy logic rules, an approach of simulating vehicle location and a decision-making procedure are adopted. Hence, models which are proper for mixed traffic can be established in DynaCHINA. For the detailed description, please see the related research works of DynaCHINA [22-25].

\section{Dynamic of Vehicle Movement}

The network consists of nodes and links with some loading elements. Each link is divided into segments that capture variations of geometry and traffic conditions along the link. Most segments are defined in advance, and additional segments can be dynamically created to capture the presence of the incidents. Nodes correspond to intersections and the loading elements are ODs.

The complexity of the flows on the network is captured by integrating three classes of models. These classes include capacities associated with roadway features; incidents and intersection controls; deterministic queuing reflecting the effect of bottlenecks; and macroscopic speed-density relationships reflecting uninterrupted flow.

3.1. Output and Acceptance Capacity. Each segment has a capacity constraint at the downstream end, referred to as the output capacity. The size of the output capacity depends on the section of the physical characteristics (such as width and slope) or unexpected events and control facilities, and so forth. Each segment is divided into a moving part and a queuing part. The moving part corresponds to the section of the segment where vehicles can move. The queuing part represents vehicles that are queued up. Vehicles are assigned to lanes according to their route to their destination. The determination of output capacities is based on recommendations from the Highway Capacity Manual [25]. When the queue length is equal to the segment length, a spillback occurs on a segment. For the detailed description, please see references $[26,27]$.

3.2. Queuing. Queuing model is a set of models, and each particular queue state (formation, disperse, obstruction, etc.) is described by different models. For example, a vehicle will join in the queue which is dissipating, and the position at the moment $(t)$ is

$$
q(t)=q(0)+l(c t-m),
$$

where $q(0)$ is the position of the end of the queue at time $t=0$. $l$ is the average length of vehicles. $m$ is the number of moving vehicles between the considered vehicle and the end of the queue at time $t=0$. That is, $m$ is the number of vehicles that reach the queue before the considered vehicle. Without loss of generality, it is assumed that the position of the upstream end of the segment is 0 . The delay of the $i$ th vehicle in the queue is given by

$$
T=\frac{i}{c}
$$

where $c$ is the output capacity of the segment.

3.3. Movement. The speed model is based on the assumption that speed is constant on the upstream section of the segment, followed by a deceleration zone covering a downstream section, where the speed of vehicles varies linearly as a function of the position.

We assume that the position of the upstream end of the segment is 0 . Therefore, the downstream end is at position $L$, where $L$ is the length of the segment. The speed function can then be written as

$$
v(z)= \begin{cases}v_{u}, & \left(0 \leq z \leq L-L_{s}\right), \\ \lambda(z-L)+v_{d}, & \left(L-L_{s}<z \leq L\right),\end{cases}
$$

where

$$
\lambda=\frac{v_{d}-v_{u}}{L_{s}}
$$

The relationship of the speed density is

$$
v_{u}= \begin{cases}v_{f}, & \rho \leq \rho_{\max }, \\ v_{f}\left[1-\left(\frac{\rho-\rho_{\max }}{\rho_{\text {jam }}}\right)^{\alpha}\right]^{\beta}, & \rho>\rho_{\max },\end{cases}
$$

where $v_{u}$ is the speed at the upstream end of the segment, $v_{a}$ is the speed at the downstream end of the segment, $L_{s}$ is the length of the deceleration zone, $v_{f}$ is the free-flow speed on the segment, $\rho_{\text {jam }}$ is the jam density, $\rho_{\max }$ is the maximum permitted density under the free flow speed, $\rho$ is the density, and $\alpha, \beta$ are parameters.

3.4. Vehicles Dynamics. For the moving parts, vehicle movement is driven by the speed density relationship model, and the vehicles are moved based on the speed [14]. As for the deceleration zone, in the absence of a queue, the vehicle will reach position $z$ at time $t(z)$, given by

$$
t(z)= \begin{cases}\frac{1}{\lambda} \log \frac{\lambda_{z+v_{u}}}{\lambda_{z_{0}+v_{u}}}, & \text { if } v_{u} \neq v_{d}, \\ \frac{z-z_{0}}{v_{u}}, & \text { if } v_{u}=v_{d},\end{cases}
$$


where at time $t=0$, a vehicle is at position $z_{0}$. Moreover, the vehicle position at any time $t$ is given by

$$
z(t)= \begin{cases}e^{\lambda t}\left(z_{0}+\frac{v_{u}}{\lambda}\right), & \text { if } v_{u} \neq v_{d}, \\ v_{u} t+z_{0}, & \text { if } v_{u}=v_{d},\end{cases}
$$

where $\lambda$ is defined by (4).

In the presence of a queue, the position $z(t)$ of a vehicle at any time $t$ is given by

$$
z(t)=e^{\lambda(t) t}\left(z_{0}+\frac{v_{u}}{\lambda(t)}\right)-\frac{v_{u}}{\lambda(t)},
$$

where

$$
\lambda(t)=\frac{-v_{u}}{q_{0}+l(c t-m)},
$$

where $q_{0}, l, c$, and $m$ are defined by (1).

3.5. Algorithm of Path Generation. For an urban road network with thousands of connections in DynaCHINA system, it is required to complete the simulation of all vehicles (maybe tens of thousands or hundreds of thousands of vehicles) in a relatively short period of 1-5 minutes, which corresponds to 30-60 minutes in the real road network. A more reasonable approach is to divide the massive complicated issue into two relatively simple issues, such as output problem of an effective path which does not have much requirement on real-time and storage and highly-effective search problem of hyperpath information. rithm.

Here is the process of generating the path collection algo-

Step 1. Set a valid path set $P=\phi$.

Step 2. Get the set of the effective paths under normal conditions and incorporate in $P: P=P \cup k-\operatorname{shortest}(G)$.

Step 3. Let the set of the temporary path $P_{h}=P$. For any path $p$ in the $P_{h}$, for all $p \in P_{h}$, we implement the following operations.

For any one edge $l$ in $p$, if $l$ is not operated, we implement the following:

(a) marked "processed",

(b) get the subgraph without the edge $l, G^{*}=G \backslash l$,

(c) generate a valid path set of $G^{*}$ and incorporate it into the $P: P=P \cup j-\operatorname{shortest}\left(G^{*}\right)$.

\section{Simulation Results}

4.1. Simulation Environment. In this simulation, we take a square format $10 \times 10$ network as the traffic network, following the references [9-11]. Two links are connected between two adjacent nodes, and each link has one direction, as shown in Figure 1. It should be pointed out that the ideal transport network is operable, because the mesoscopic traffic flow

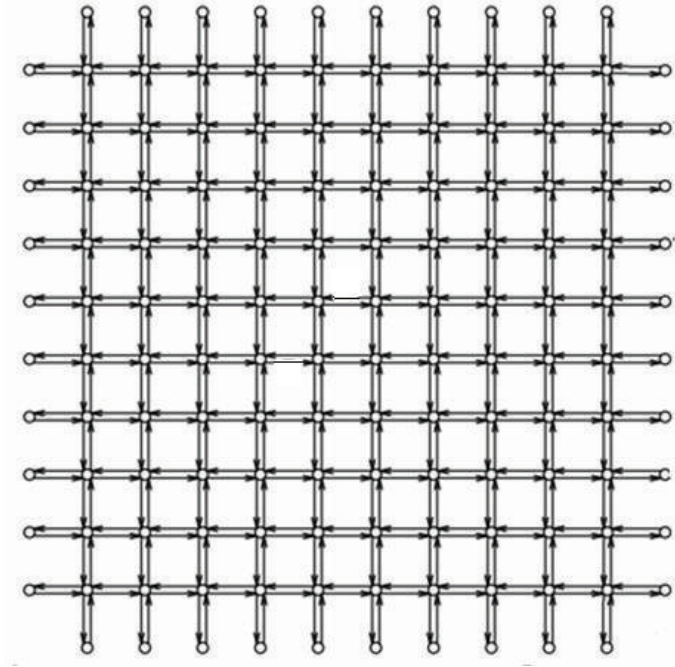

FIGURE 1: $10 \times 10$ bidirectional grid network.

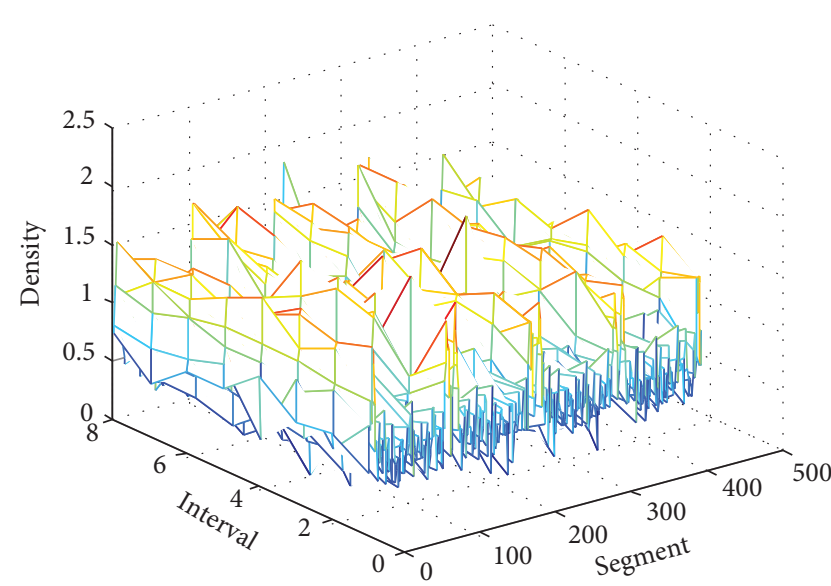

Figure 2: Density on each segment per interval when demand is 1.

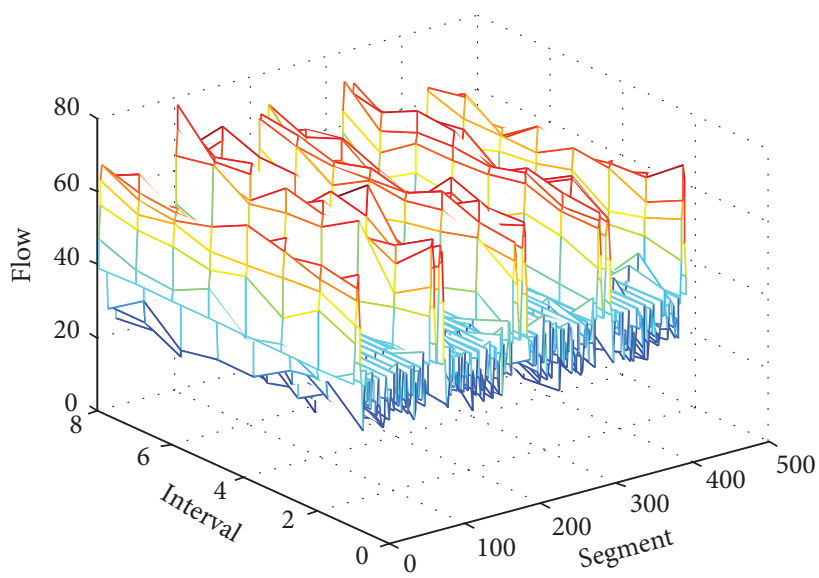

FIgURE 3: Flow on each segment per interval when demand is 1. 


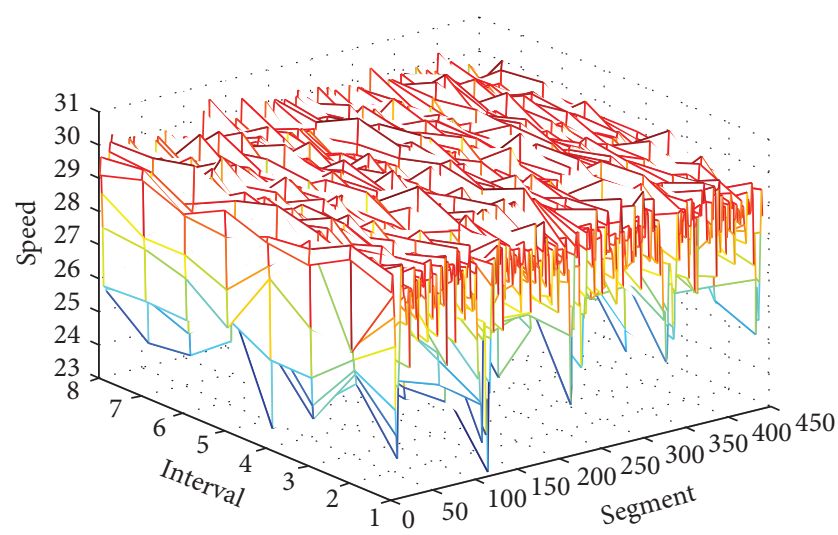

FIgURE 4: Speed on each segment per interval when demand is 1.

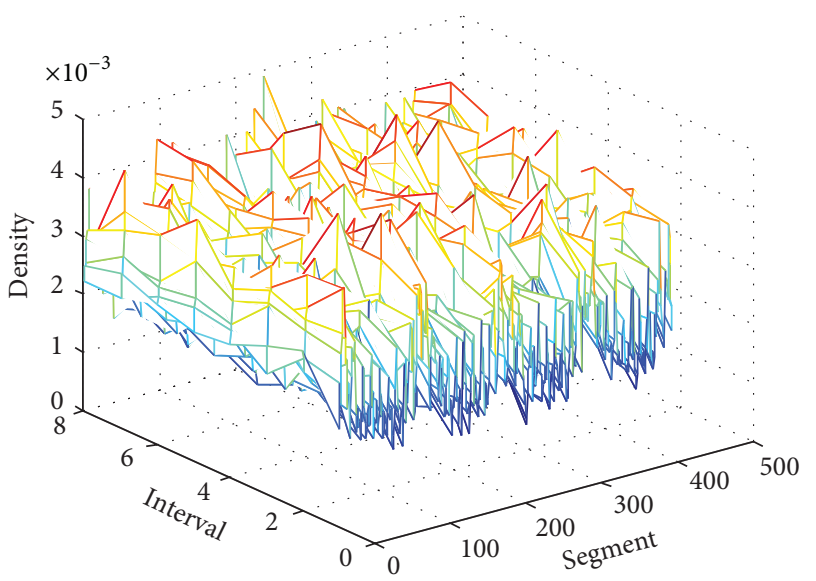

Figure 5: Density on each segment per interval when demand is 3.

model focuses on the vehicle group behavior, and the road network structure has little effect on the content of this study. Each link is defined as 500 meters uniformly, the default is two lanes, and every section is defined as one segment. Each edge node is defined as the transportation zone (i.e., OD points). Jam density is defined as $0.1150 \mathrm{pcu} / \mathrm{m} / \mathrm{L}, \alpha$ is $1.9420, \beta$ is 0.5040 , and the input and output capacity of segment is $0.611 \mathrm{v} / \mathrm{s}$. Without signal control, the designed maximum speed is $60 \mathrm{~km} / \mathrm{h}$ at all intersections. Simulation time is defined as $15 \mathrm{~min}$, and 8 time periods are considered, leading to a total of two hours of simulation. Network OD demand is loaded with periodic closure boundary condition.

In this paper, the anisotropic mesoscopic simulation modeling concept and model are employed. This is a feature different from other software. The anisotropic mesoscopic simulation modeling concept is defined by the following: for any vehicle $i$, its those leading vehicles (in the same lane or in the adjacent lanes) present in vehicle $i$ 's immediate downstream and within a certain distance are considered to influence vehicle is speed response. By a simple test, at each level of demand, the output average network speed by using DynaCHINA is higher than that using DynaMIT or DynaSMART.

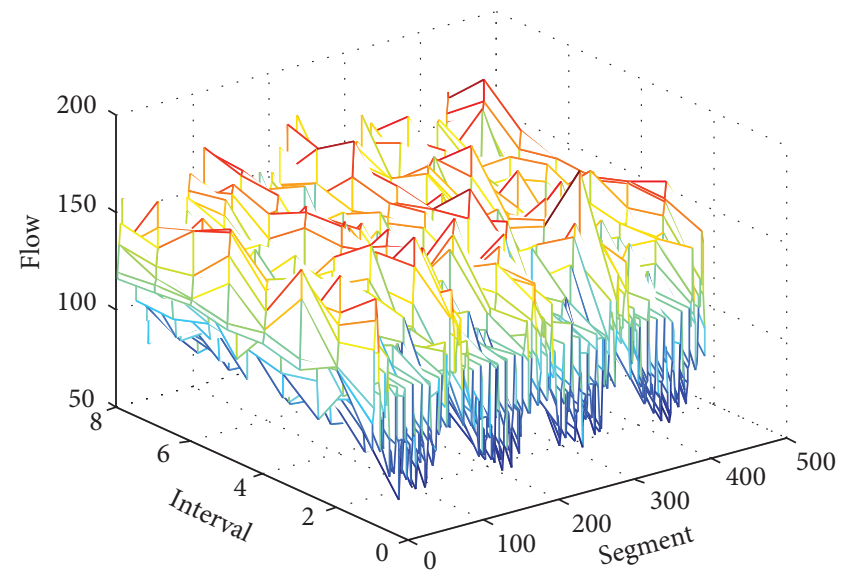

FIGURE 6: Flow on each segment per interval when demand is 3.

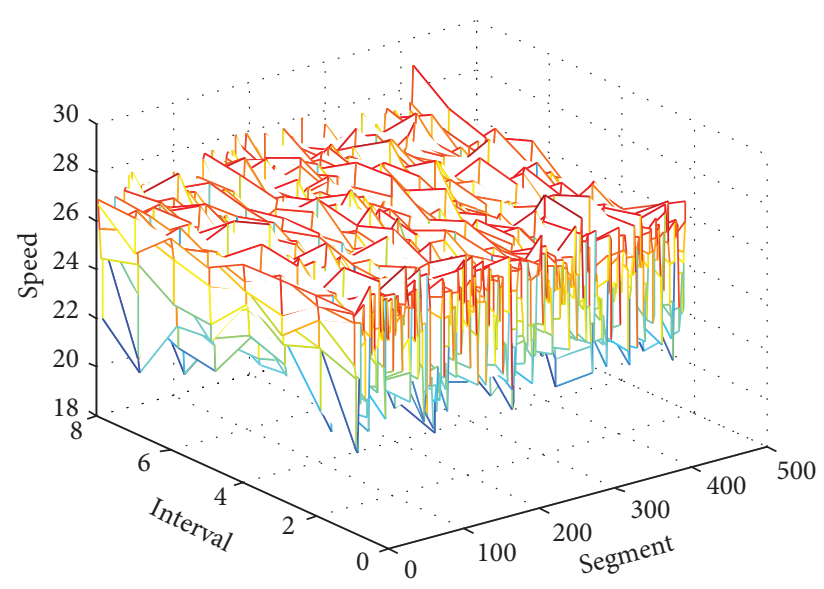

FIGURE 7: Speed on each segment per interval when demand is 3.

4.2. Performance for the Network at Different Demand Level. Generally, the jam density on the segment can be regarded as a benchmark, which is divided into five equally parts, denoted as very smooth, smooth, congestion, very congestion, and jam, respectively. If there are most of links in the network having the same level of jam density, the network can be considered to have the same traffic state.

When the demand of all the OD pairs is set to be 1 , the network has not the phenomenon of congestion, which belongs to very smooth. At this demand level, the max vehicle number loaded on the network is 1207 . We can see the average density, speed, and flow on each segment at every interval, as shown in Figures 2-4.

When the demand of all the OD pairs is 3 , the congestion occurs in some segments, as shown in Figures 6 and 7. The number of vehicles loaded on the network is 2867 . Since most segments in this case are not congested, the state of network belongs to smooth.

As can be seen from Figures 2, 3, 4, 5, 6, and 7, the average density and flow are increased very much, but the average speed has some decreased.

When the demand of all the OD pairs is set to be 5 , the state of network becomes worse rapidly, which belongs to 


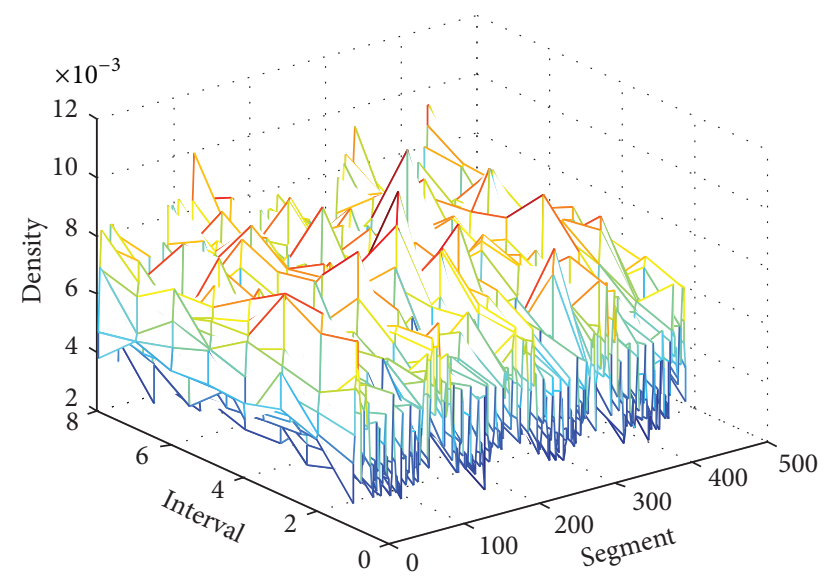

FIGURE 8: Density on each segment per interval when demand is 5.

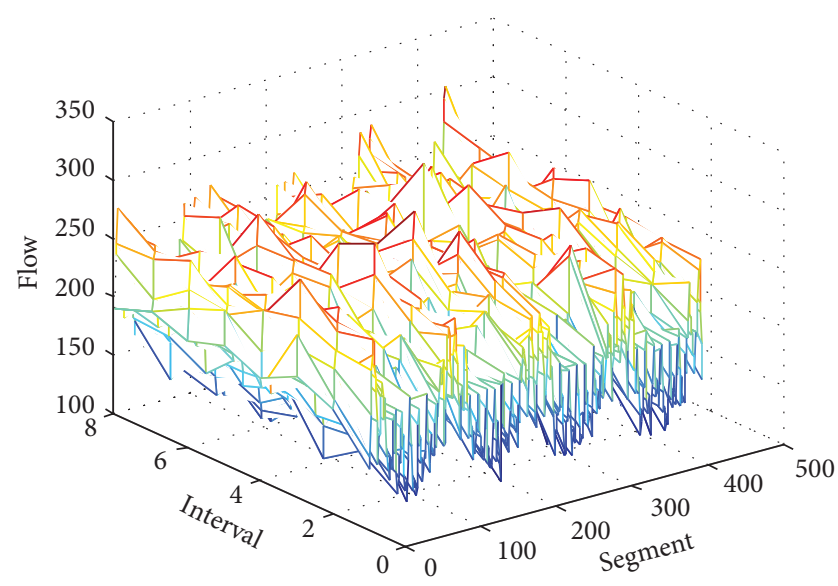

FIGURE 9: Flow on each segment per interval when demand is 5.

very congestion, almost jam state, as shown in Figures 8, 9, and 10. The max number of vehicles loaded on the network is 4386. Most segments are congested in this case. The average density and average flow on the segment become increased sharply, and the average speed is decreased smoothly. If we keep increasing the demand, the network condition becomes worse and worse, leading to the state of extreme congestion. When the demand becomes 1 , the loaded vehicle is increased by 780 , since we have 40 OD pairs. Another interesting finding is that no matter what the demand is the moving vehicles loaded on the network form a center of symmetry. This is because the OD points are symmetry and the demand is the same in the simulations. However, the kinematic characteristic of vehicles is changeless, and the essential changes for the law of vehicle motion will not occur.

\section{Concluding Remarks}

In this paper, the traffic states and traffic congestion propagation have been studied by developing an improved mesoscopic traffic flow model in the dynamic software of DynaCHINA. The average density, flow, and speed on the segment at every interval for different demand levels are

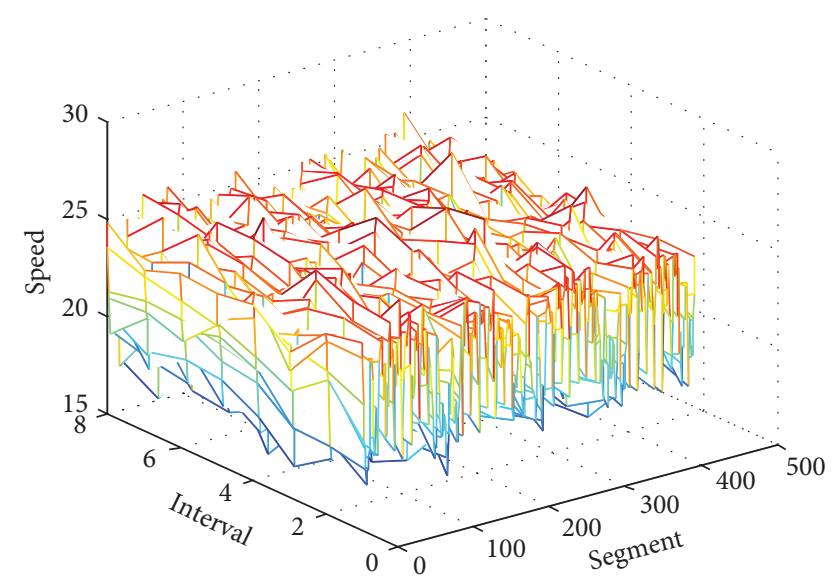

FIGURE 10: Speed on each segment per interval when demand is 5.

mainly investigated. Simulation results show that the proposed model and method are capable of capturing the real traffic state. Since the simulations are implemented in a bidirectional grid network which should be improved in the future, the congestion propagation characteristics in the traffic accident should be paid more attention.

\section{Acknowledgments}

This work is partially supported by National Basic Research Program of China (2012CB725400), National Natural Science Foundation of China (71171124, 71131001, and 71222101), Joint Science \& Technology of the Ministry of Transport Industry (2009-353-337-480), and Super Computing Technology Special Project of Shandong Province (2011YD01104).

\section{References}

[1] C. F. Daganzo and J. A. Laval, "Moving bottlenecks: a numerical method that converges in flows," Transportation Research B, vol. 39, no. 9, pp. 855-863, 2005.

[2] G. F. Newell, "A simplified theory of kinematic waves in highway traffic-part III: multi-destination flows," Transportation Research B, vol. 27, no. 4, pp. 305-313, 1993.

[3] D. Ni and J. D. Leonard II, "A simplified kinematic wave model at a merge bottleneck," Applied Mathematical Modelling, vol. 29, no. 11, pp. 1054-1072, 2005.

[4] G. Ziyou and S. Yifan, "A reserve capacity model of optimal signal control with user-equilibrium route choice," Transportation Research B, vol. 36, no. 4, pp. 313-323, 2002.

[5] G. Gentile, L. Meschini, and N. Papola, "Spillback congestion in dynamic traffic assignment: a macroscopic flow model with time-varying bottlenecks," Transportation Research B, vol. 41, no. 10, pp. 1114-1138, 2007.

[6] W. H. K. Lam and Y. Yin, "An activity-based time-dependent traffic assignment model," Transportation Research B, vol. 35, no. 6, pp. 549-574, 2001.

[7] H. Guo, W. Wang, W. Guo, X. Jiang, and H. Bubb, "Reliability analysis of pedestrian safety crossing in urban traffic environment," Safety Science, vol. 50, no. 4, pp. 968-973, 2012.

[8] W. H. Wang, W. Zhang, H. W. Guo, H. Bubb, and K. Ikeuchi, "A behavioural car-following safety model for microscopic 
simulation of traffic flow with various driving characteristics," Transportation Research C, vol. 19, no. 6, pp. 1202-1214, 2011.

[9] J. C. Long, Z. Y. Gao, H. L. Ren, and A. p. Lian, "Urban traffic congestion propagation and bottleneck identification," Science in China F, vol. 55, no. 7, pp. 948-964, 2008.

[10] J. C. Long, Z. Y. Gao, P. Orenstein, and H. L. Ren, "Control strategies for dispersing incident-based traffic jams in two-way grid networks," IEEE Transactions on Intelligent Transportation Systems, vol. 13, no. 2, pp. 469-481, 2011.

[11] A. Zhang, Z. Gao, and H. Ren, "Incident-based traffic congestion control strategy," Science China Technological Sciences, vol. 54, no. 5, pp. 1338-1344, 2011.

[12] P. Roberg, "Development and dispersal of area-wide traffic jams," Traffic Engineering \& Control, vol. 35, no. 6, pp. 379-386, 1994.

[13] P. Roberg, "A distributed strategy for eliminating incidentbased traffic jams from urban networks," Traffic Engineering \& Control, vol. 36, no. 6, pp. 348-355, 1995.

[14] P. Roberg and C. R. Abbess, "Diagnosis and treatment of congestion in central urban areas," European Journal of Operational Research, vol. 104, no. 1, pp. 218-230, 1998.

[15] P. Roberg-Orenstein, C. R. Abbess, and C. Wright, “Traffic jam simulation," Journal of Maps, pp. 107-121, 2007.

[16] C. Wright and P. Roberg, "The conceptual structure of traffic jams," Transport Policy, vol. 5, no. 1, pp. 23-35, 1998.

[17] C. Wright and P. Roberg-Orenstein, "Simple models for traffic jams and congestion control," Proceedings of the Institution of Civil Engineers, vol. 135, no. 3, pp. 123-130, 1999.

[18] M. Ben-Akiva, M. Bierlaire, D. Burton, H. N. Koutsopoulos, and R. Mishalani, "Network state estimation and prediction for realtime transportation management applications," Networks and Spatial Economics, vol. 1, no. 3-4, pp. 293-318, 2001.

[19] H. S. Mahmassani, "Dynamic network traffic assignment and simulation methodology for advanced systems management applications," Networks and Spatial Economics, vol. 1, no. 3-4, pp. 267-292, 2001.

[20] C. Antoniou, M. Ben-Akiva, and H. N. Koutsopoulos, "Dynamic traffic demand prediction using conventional and emerging data sources," IEE Proceedings, vol. 153, no. 1, pp. 97-104, 2006.

[21] T. G. Oketch, "New modeling approach for mixed-traffic streams with nonmotorized vehicles," Transportation Research Record, no. 1705, pp. 61-69, 2000.

[22] Y. Lin and H. B. Song, "DynaCHINA: specially built real time traffic prediction system for China," in Proceedings of the 86th Annual Transportation Research Board Meeting, Washington, DC, USA, 2007.

[23] S.-B. Li, Z.-Y. Gao, Y. Lin et al., "Real-time path searching algorithm for large traffic network," Journal of Transportation Systems Engineering and Information Technology, vol. 9, no. 5, pp. 141-147, 2009.

[24] S. B. Li, J. J. Wu, Z. Y. Gao, Y. Lin, and B. B. Fu, "Bi-dynamic analysis of traffic congestion and propagation based on complex network," Acta Physica Sinica, vol. 60, no. 5, Article ID 050701, 2011.

[25] Highway Capacity Manual, 1994.

[26] M. Ben-Akiva, "Development of a deployable real-time dynamic traffic assignment system," Evaluation Report (Part A): Evaluation of Estimation and Prediction Capabilities, Massachusetts Institute of Technology, 2003.

[27] M. Ben-Akiva, "Development of a deployable real-time dynamic traffic assignment system," Evaluation Report (Part B):
Planning Functionality (DynaMIT-P) and Applications, Massachusetts Institute of Technology, 2003. 


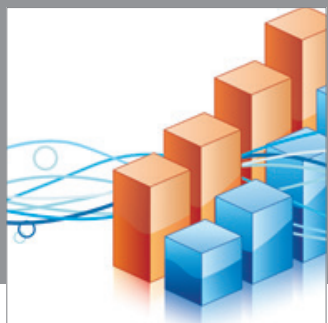

Advances in

Operations Research

mansans

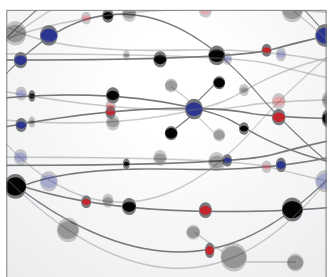

The Scientific World Journal
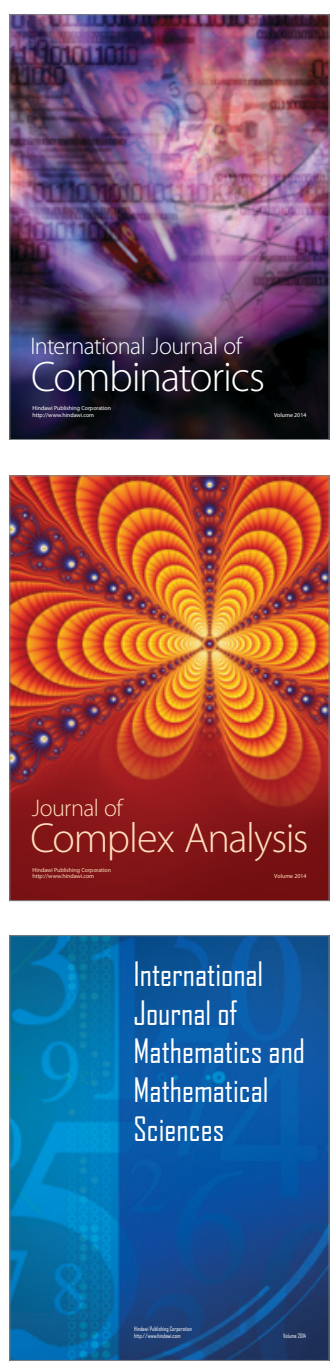
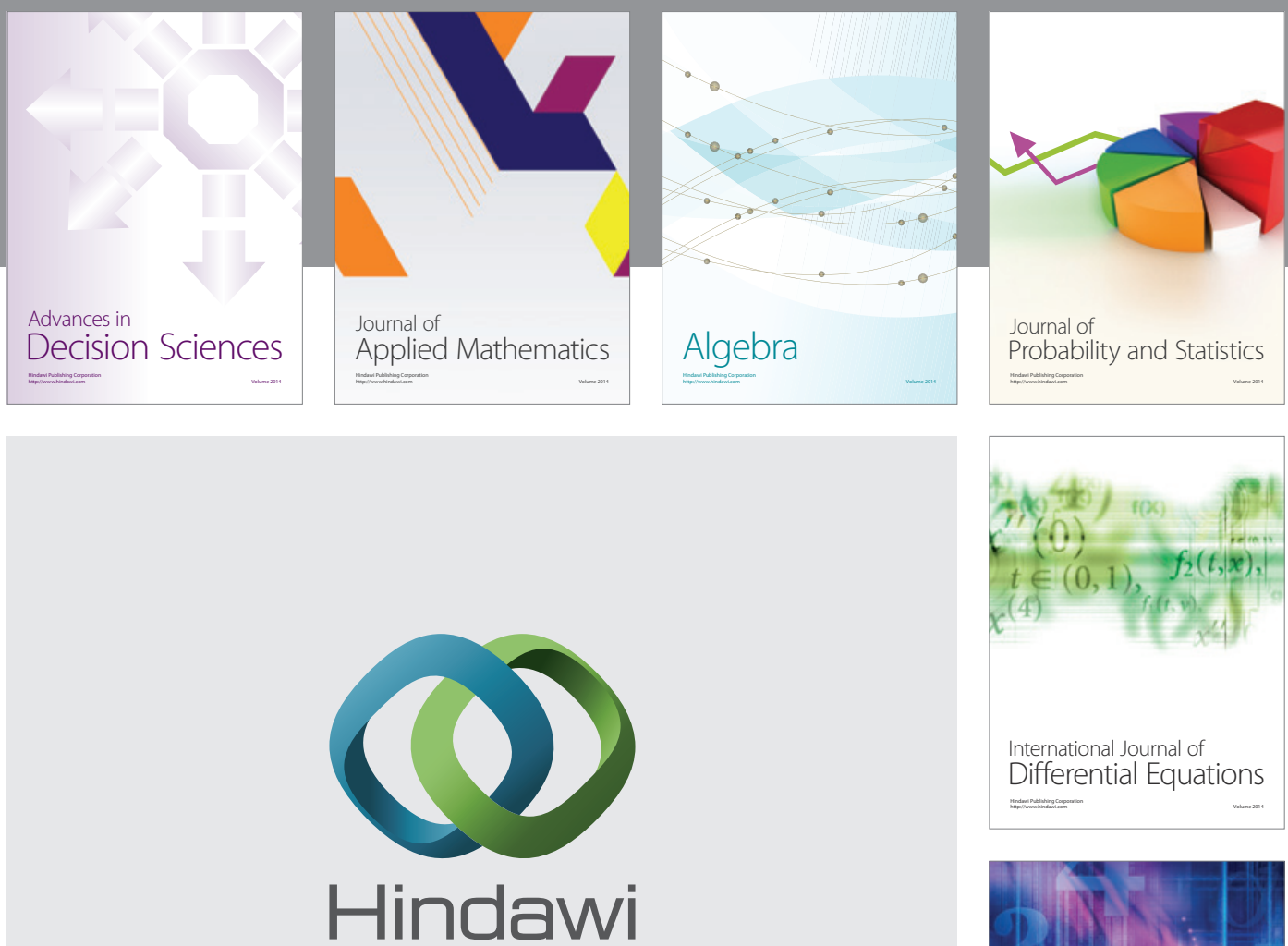

Submit your manuscripts at http://www.hindawi.com
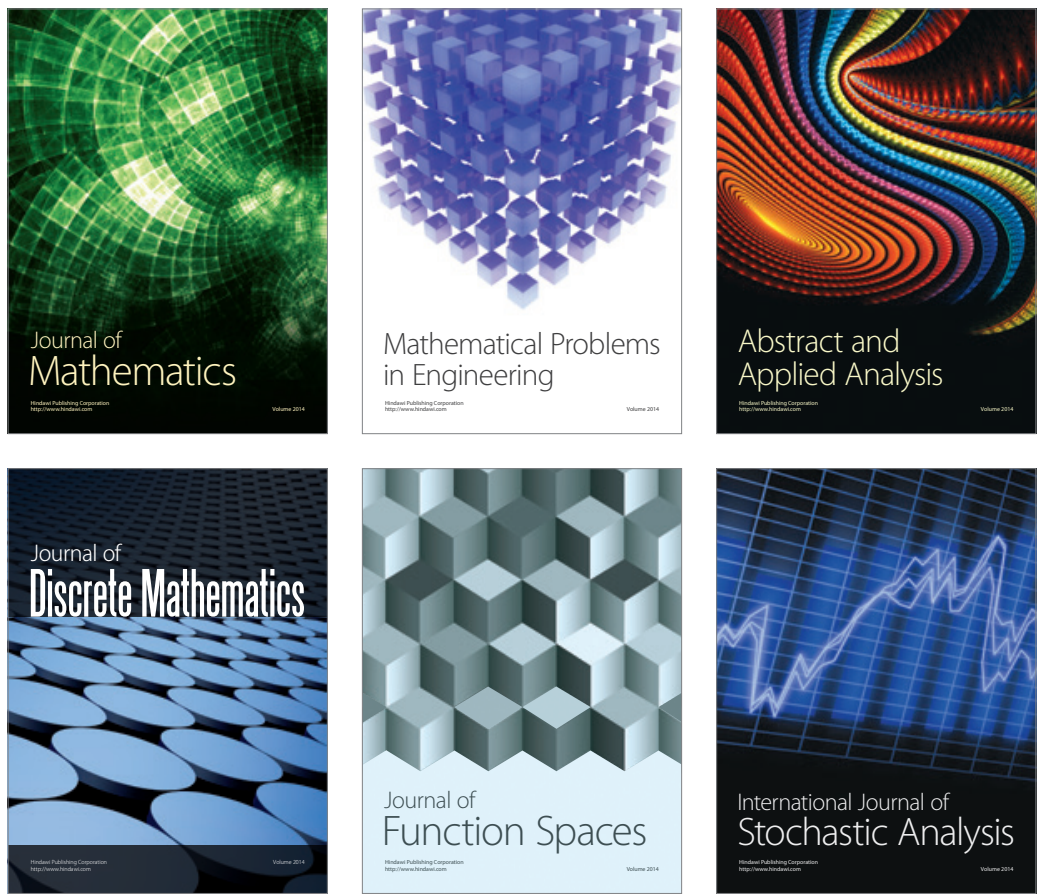

Journal of

Function Spaces

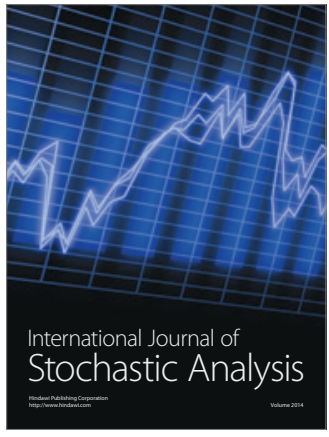

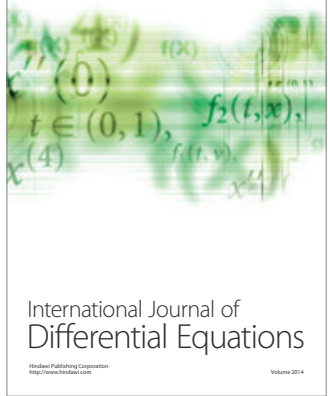
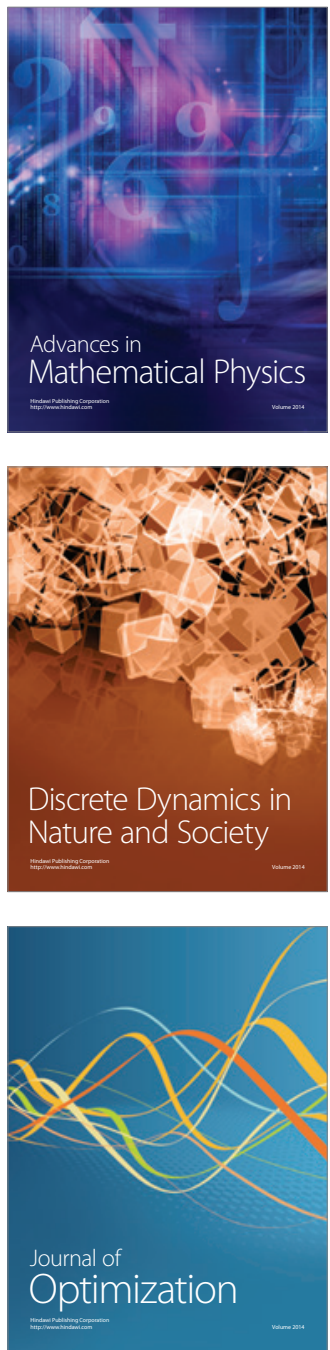